\title{
Massive carbon dioxide embolism during pneumoperitoneum for laparoscopic adrenalectomy: A case report
}

\author{
Hsiao-Chun Teng ${ }^{1 *}$, Hui-Min Yeh ${ }^{1}$, Shi-Meng Wang ${ }^{2}$ and NAI-SIN JI ${ }^{3}$ \\ ${ }^{1}$ Department of Anesthesiology, National Taiwan University Hospital, Taipei, Taiwan \\ ${ }^{2}$ Department of Urology, National Taiwan University Hospital, Taipei, Taiwan \\ ${ }^{3}$ Department of Cardiovascular surgery, National Taiwan University Hospital, Taipei, Taiwan
}

\begin{abstract}
A 65-year-old man, with suspected right adrenal metastasis from hepatic carcinoma, was scheduled to undergo a laparoscopic right adrenalectomy. About 75 minutes into the operation, when attempting to remove the adrenal gland that was tightly adhesive to the IVC, the surgeon accidently nicked the large inferior vena cava which resulted in a (IVC) tear of about $2 \times 2 \mathrm{~cm}$. Considering the difficulty of performing exploratory laparotomy due to previous hepatic tumor excision related intraabdominal adhesion, the surgeon tried to sow the torn IVC through the use of the laparoscope and to control the bleeding via carbon dioxide (CO2) insufflation pressure. This resulted in a massive amount of gas entering the vessel via the IVC broken hole. When we performed a transesophageal echocardiography(TEE), it was revealed that the right atrium and right ventricle were totally white out because they were full of air (Figure 1). About 20 minutes after the IVC tear, the TEE image also revealed that the left heart also contained scattered air embolisms (Figure 2). The surgeon spent about 60 minutes on repairing the IVC tear. Blood pressure was stable during the IVC tear insult but dropped slightly after the IVC tear repaired. Low dose norepinephrine and dopamine continuous infusion were administered to stabilize the patient's blood pressure. After the operation, the patient presented mild weakness in his left limbs. Fortunately, the symptoms dissipated two days after the surgery. 12 days later, the patient was discharged without any neurologic sequelae.
\end{abstract}

\section{Introduction}

Laparoscopy is deemed a "minimally" invasive procedure that allows for exploration through an endoscope of the peritoneal cavity after insufflation of carbon dioxide $\left(\mathrm{CO}_{2}\right)$ [1]. It has been widely used during many types of surgery. There are a number of advantages compared to open procedure which includes: decreased risk of bleeding, infection, and postoperative pain6. However, there are also some complications associated with laparoscopic surgery. For example, the pneumoperitoneum may lead to decreased cardiac output and/or increased systemic vascular resistance. In addition, the Carbon dioxide pneumoperitoneum may cause respiratory acidosis and deep venous thrombosis [2].

Although $\mathrm{CO}_{2}$ embolisms are rare, they are potentially fetal if they are not diagnosed or treated immediately. This is especially true when the $\mathrm{CO}_{2}$ from the right heart flows into the left heart [3] Clinically, carbon dioxide embolism can present itself with systemic hypotension, dyspnea, cyanosis, tachycardia or bradycardia, arrhythmia or asystole, elevated pulmonary arterial pressure(PAP), elevated central venous pressure, hypoxemia and increased arterial partial pressure of carbon dioxide, increase or decrease end-tidal carbon dioxide tension [4]. There are case reports about cardiac arrest associated with $\mathrm{CO} 2$ embolism during laparoscopic surgery8. We are reporting this case of potentially fatal complication of massive $\mathrm{CO} 2$ embolism during laparoscope adrenalectomy at our institute. Even the patient had experienced a massive $\mathrm{CO} 2$ embolism during the surgery, he discharged without any complication by the twelfth day after the operation.

\section{Case report}

A 65-year-old man, suspected with right adrenal metastasis, was scheduled to undergo a laparoscopic right adrenalectomy. The patient presented with a history of hepatocellular carcinoma, BCLC stage A. He underwent a hepatic tumor excision 2 years ago, with repeated recurrence. The recurrence hepatic tumors were treated with RFA and TACE. However, following computed tomography scan 3 months ago found suspected right adrenal metastasis. The doctor suggested him to receive a laparoscopic right adrenalectomy. The patient is $167 \mathrm{~cm}$ tall and weighed $67 \mathrm{~kg}$. His physical examination revealed clear breathing sounds to auscultation and regular heart rate with normal resting blood pressure (112/64 mmHg).

The patient's preoperative stats in the operation room were: blood pressure $141 / 69 \mathrm{mmHg}$; pulse $86 / \mathrm{min} ; \mathrm{SaO}_{2} \%$ on room air $99 \%$. The patient was preoxygenated with

$5 \mathrm{~L}$ of oxygen via face mask, and underwent induction with $100 \mu \mathrm{g}$ of fentanyl, $0.2 \mathrm{mg}$ of glycopyrolate, $250 \mathrm{mg}$ of Citosol, $30 \mathrm{mg}$ of propofol and $10 \mathrm{mg}$ of cisatracurium IV. Ventilation was set at a tidal volume

Correspondence to: Huei-Ming Yeh, MD, Department of Anesthesiology, National Taiwan University Hospital, Taipei, Taiwan, no 7 Chung-Shan South Road, Taipei, Taiwan ROC, Tel:886-2-2312-3456 extension 65512: Fax: 886-22341-1529; E-mail: y.y.hhmm@gmail.com

Received: December 30, 2016; Accepted: February 03, 2017; Published: February 07, 2017 
of $550 \mathrm{ml}$ at the rate of $10-12 / \mathrm{min}$ and with $\mathrm{FiO}_{2} 40-60 \%$. Desflurane was administered to maintain a Minimum Alveolar Concentration (MAC) of 1.0 to 1.2 in combination with $0.5 \mathrm{~L}$ of oxygen and $0.5 \mathrm{~L}$ of air. After anesthesia, an arterial catheter was inserted into the patient to continuously monitor the patient's arterial pressure.

The patient was positioned in a left lateral recumbent position. An additional $50 \mu \mathrm{g}$ fentanyl was administered while the Veress needle was introduced. During the surgery, we began with a high flow of $\mathrm{CO}_{2}$ intra-peritoneal insufflation to achieve an intra-abdominal pressure of $15 \mathrm{mmHg}$.

During the initial 75 minutes, the patient's vital signs were stable and the procedure was performed smoothly. However, when attempting to remove the adrenal gland and adrenal tumor which was tightly adhered to the IVC, the surgeon accidently nicked the large inferior vena cava which resulted in a large IVC tear of about $2 \times 2 \mathrm{~cm}$. The bleeding from the torn IVC was temporary compressed by the guaze. However, we suspect that a massive amount of $\mathrm{CO}_{2}$ entered the vessel via the IVC broken hole. Taking into account the difficulty of performing exploratory laparotomy due to previous hepatic tumor excision related intra-abdominal adhesion, the surgeon tried to sow the torn IVC by the use of laparoscope and to control the bleeding via carbon dioxide $\left(\mathrm{CO}_{2}\right)$ insufflation pressure. However, we suspect that a massive amount of $\mathrm{CO}_{2}$ entered the vessel via the IVC broken hole while the surgeon sow the torn IVC. They spent about 60 minutes on repairing the IVC tear. Blood pressure was stable during the IVC tear insult but dropped slightly after the torn IVC repaired. Systolic blood pressure during the insult was kept at 100 130 $\mathrm{mmHg}$, pulse $70 \sim 90 / \mathrm{min}$, airway pressure was maintained at $35 \mathrm{mmHg}$ which may be induced by the laparoscope and SPO $2 \%$ was $100 \%$. However, the end tidal $\mathrm{CO}_{2}$ gradually elevated from $35 \mathrm{mmHg}$ to $53 \mathrm{mmHg}$. The ventilator respiratory rate was then set higher to wash out $\mathrm{CO}_{2}$.

We then inserted TEE to evaluate the severity of the $\mathrm{CO}_{2}$ embolism while the surgeon tried to fix the IVC tear. Through the TEE image, we noticed that the right atrium and right ventricle were totally white out and full of air (Figure 1). About 20 minutes after IVC tear, the TEE image also revealed that the left heart also had scattered air embolisms (Figure 2). We then inserted a CVC via the right internal jugular vein, to attempt to drain the $\mathrm{CO}_{2}$ through the CVC, which failed. Sewing the

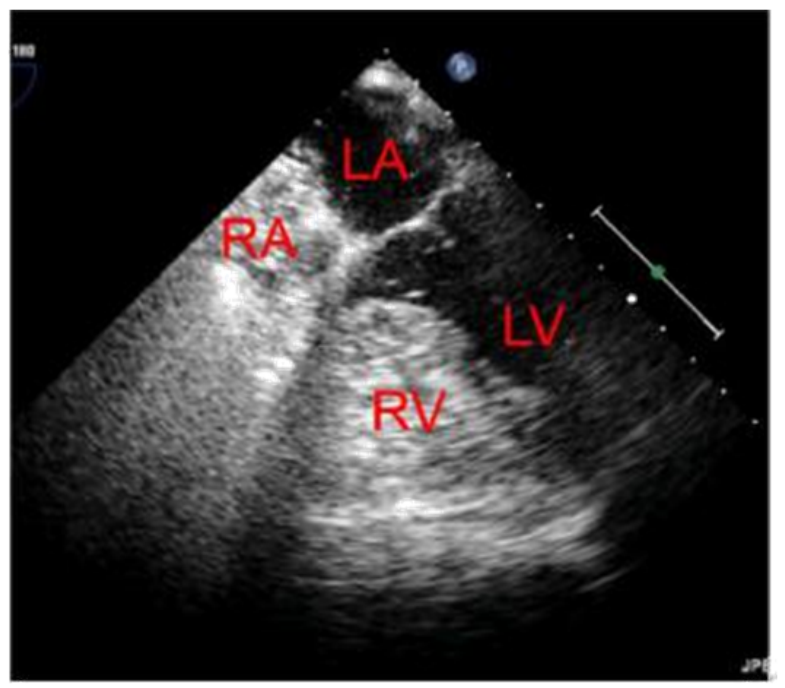

Figure 1. TEE four chamber view. RA and RV were full of air $\left(\mathrm{CO}_{2}\right)$, totally white out persisted for 60 minutes

RA: right atrium; RV: right ventricle; LA: left atrium; LV: left ventricle

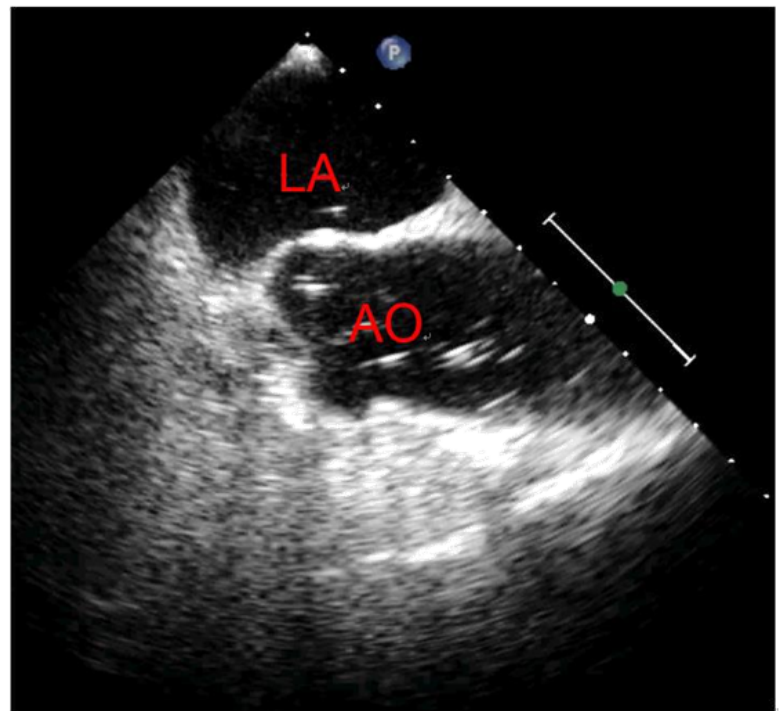

Figure 2. TEE long axis view. $\mathrm{CO}_{2}$ emboli can be found even in the ascending aorta LA: left atrium; AO: ascending aorta

fragile IVC tissue proved difficult, so we consulted cardiac surgeons who were then able to repair the torn IVC. The total massive $\mathrm{CO}_{2}$ influx

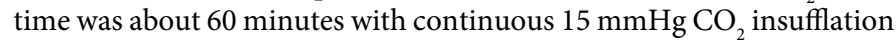
pressure. And the total blood loss was about $1250 \mathrm{ml}$.

The drop-in blood pressure caused by the IVC tear was restored by low dose norepinephrine intermittent bolus $(5 \mathrm{mcg})$ and blood transfusion. In total, we infused $2 \mathrm{u}$ of pRBC, $2 \mathrm{u}$ of whole blood and $12 \mathrm{u}$ of platelet. The blood gas $1 \mathrm{hr}$ after the insult showed respiratory acidosis with $\mathrm{P}^{\mathrm{H}} 7.01, \mathrm{PCO}_{2} 129 \%, \mathrm{PO}_{2} 75 \%\left(\mathrm{FiO}_{2} 86 \%\right), \mathrm{SaO}_{2} 83 \%$. Pulmonary $\mathrm{CO}_{2}$ embolism induced hypercapnia and hypoxemia was under impressed. We suspected pulmonary edema because of the presence of pink bubbled sputum. $\mathrm{SPO}_{2} \%$ was also gradually decreased to $93 \%$. The blood gas 3 hours after the insult showed $\mathrm{PH} 7.1, \mathrm{PCO}_{2}$ $82.9 \%$, $\mathrm{PO} 2186 \%$ ( $\mathrm{FiO}_{2} 85 \%$ ), $\mathrm{SaO}_{2} 99 \%$. Post-surgery, the patient was transferred to the ICU with mechanical ventilation to receive further care.

During the ICU admission, the ECG showed S1Q3 without ST-T change. Acute respiratory distress syndrome (ARDS) was also diagnosed via chest radiography and clinical presentation. Low dose norepinephrine and dopamine continuous infusion were administered for SIRS reaction but were then gradually tapered off in 2 days. Neurological examinations showed mild weakness with muscle power of $4+$ in the patient's left limbs during the first day after the surgery but these symptoms dissipated by the second day after the operation. By the second day the patient was under a stable enough condition to be extubated and transferred out of the ICU into the general ward by the fifth day. He was discharged by the twelfth day after the surgery without any residual complication.

\section{Discussion}

Carbon dioxide is the most widely used insufflation gas. Most serious cases of $\mathrm{CO}_{2}$ embolism reported in the literature occurs during the beginning of the procedure, usually due to the misplacement of the Veress needle either directly into a vein or parenchymal organ4. Lesser amounts of $\mathrm{CO}_{2}$ may also enter circulation through openings in injured vessels, as seen in our case, which explains the late onset of the carbon dioxide embolisms [4]. 
Rapid entrainment of a large volume of gas can lead to the formation of large emboli, which may lodge in a large central vessel and potentially lead to cardiovascular collapse [5]. In our case, the surgeon was facing the dilemma of whether to transform into open surgery. Considering previous hepatic tumor excision related intra-abdominal adhesion, exploratory laparotomy to approach the bleeder would be also difficult [6]. Instead, we tried an adventurous method: we controlled the IVC bleeding via the carbon dioxide $\left(\mathrm{CO}_{2}\right)$ insufflation pressure, at the same time, the surgeon sow the torn IVC by the use of laparoscope. A necessary condition for the development of gas embolism is the presence of an open vein with a lower pressure in the vein than the surrounding pressure [7]. Nevertheless, it is because the little higher pressure of the $\mathrm{CO}_{2}$ gas insufflation, the bleeding rate was not so fast to be life-threatening. But the pressure of $\mathrm{CO}_{2}$ gas insufflation can't be too high to make fetal amount of $\mathrm{CO}_{2}$ embolism.

Fortunately, our patient did not develop cardiovascular collapse even encounter this massive $\mathrm{CO}_{2}$ embolism. It may because the slow entrainment of the $\mathrm{CO}_{2}$ and the high solubility of $\mathrm{CO}_{2}$ (20:1 to oxygen and 25:1 to nitrogen) [8]. Mayer, et al. described a mortality of $60 \%$ at a continuous intravenous $\mathrm{CO}_{2}$ infusion rate of $1.2 \mathrm{~mL} / \mathrm{kg} / \mathrm{min}$, which is equivalent to a rate of $72 \mathrm{~mL} / \mathrm{min}$ for a $60 \mathrm{~kg}$ person. That volume is only $5 \%$ of the volume of carbon dioxide that may be infused into a vein, intentionally cannulated by a Veres needle, in one minute at a low-flow rate [9]. Our effort to keep the balance of IVC venous pressure and surrounding $\mathrm{CO}_{2}$ insufflation pressure during the episode may prevent a massive fetal $\mathrm{CO}_{2}$ embolism and a fetal bleeding rate.

About 20 minutes after IVC tear, the TEE image also revealed scattered air embolisms in the left heart. The foramen ovale didn't open on the TEE image. We suspect that the left heart gas emboli may have been caused from pulmonary capillary diffusion when large amounts of air entered the vena cava. Post-surgery, the patient developed neurologic signs of left limbs weakness but was able to rapidly recover in 2 days. The rapid improvement maybe attributed to the high solubility of $\mathrm{CO}_{2}$.

Treatment of $\mathrm{CO}_{2}$ embolisms consists of the immediate cessation of insufflation and the release of the pneumoperitoneum, steep headdown and left lateral decubitus position. And the discontinuation of nitrous oxide and to ventilate with $100 \%$ oxygen. In addition, adequate hydration and the placement of a CVC to aspirate $\mathrm{CO}_{2}$ is required. Our patient was already in the head-down and left lateral decubitus position as it was required for surgery. However, the insufflation of $\mathrm{CO}_{2}$ didn't cease in our patient, because the surgeon needed to control the bleeding through applying pneumoperitoneum pressure.

In conclusion, our patient's prognosis was good despite having gone through a massive $\mathrm{CO}_{2}$ embolism event. The patient's relatively stable vital signs and possibly intra-abdominal adhesion were the reasons why we did not transfer the laparoscope to exploratory laparotomy nor immediately cease $\mathrm{CO}_{2}$ insufflation. In addition, the rapid improvement from the neurological symptoms maybe attribute to the high solubility of $\mathrm{CO}_{2}$. Hence, we hypothesize that the decision of whether to cease the pneumoperitoneum immediately when $\mathrm{CO}_{2}$ embolisms occur, may depend on the patient's hemodynamics. In admission, it is important to find the balance between venous and $\mathrm{CO}_{2}$ insufflation pressure to avoid massive bleeding or fetal amount of $\mathrm{CO}_{2}$ embolisms. And that the neurological tolerance of $\mathrm{CO}_{2}$ emboli may be much higher than that of other gases.

\section{References}

1. Yao F-SF, Malhotra V, Fontes ML, eds. (2008) Yao and Artusio's Anesthesiology Problem-Oriented Patient Management. 6th ed. Philadelphia, PA: Lip-pincott Williams \& Wilkins pp875-877.

2. Richard A Perugini and Mark P Callery (2001) Complications of laparoscopic surgery. Surgery Treatment evidence-based and problem-oriented. Holzheimer RG, Mannick JA. [Crossref]

3. Uchida S, Yamamoto M, Masaoka Y, Mikouchi H, Nishizaki Y (1999) A case of acute pulmonary embolism and acute myocardial infarction with suspected paradoxical embolism after laparoscopic surgery. Heart Vessels 14: 197-200. [Crossref]

4. Park EY, Kwon JY, Kim KJ (2012) Carbon dioxide embolism during laparoscopic surgery. Yonsei Med J 53: 459-466. [Crossref]

5. Amir Abu Zikry, Kalindi DeSousa, Khaled H Alanezi (2011) Carbon dioxide embolism during laparoscopic sleeve gastrectomy. J Anaesthesiol Clin Pharmacol 27: 262-265. [Crossref]

6. Hu J, Or BH, Hu K, Wang ML (2016) Comparison of the post-operative outcomes and survival of laparoscopic versus open resections for gastric gastrointestinal stromal tumors: A multi-center prospective cohort study. Int J Surg [Crossref]

7. Derouin M, Couture P, Boudreault D, Girard D, Gravel D (1996) Detection of gas embolism by transesophageal echocardiography during laparoscopic cholecystectomy. Anesth Analg 82: 119-121. [Crossref]

8. II-SeoK Kim, Jae-Woo Jung, Keum-Man Shin (2012) Cardiac arrest associated with carbon dioxide gas embolism during laparoscopic surgery for colorectal cancer and liver metastasis -A case report. Korean J Anesthesiol 63: 469-472

9. Mayer KL1, Ho HS, Mathiesen KA, Wolfe BM (1998) Cardiopulmonary responses to experimental venous carbon dioxide embolism. Surg Endosc 12: 1025-1030. [Crossref]

Copyright: (C2017 Teng HC. This is an open-access article distributed under the terms of the Creative Commons Attribution License, which permits unrestricted use, distribution, and reproduction in any medium, provided the original author and source are credited. 